\title{
Simulation analysis for load test of Chang pugang Bridge
}

\author{
Lanlin Zou ${ }^{1, a}$, Yaohui $\mathrm{Wu}^{2, \mathrm{~b}}$ \\ ${ }^{1}$ College of Automobile and Traffic Engineering, Wuhan University of Science and Technology, \\ Wuhan, China 430081 \\ a13971206577@163.com, ${ }^{\mathrm{b}}$ 1738884602@qq.com,
}

Keywords: loading test; simulation analysis; experimental condition

Abstract. According to Extension project construction drawing design of Chang pugang Bridge and some related files, we built the mathematical model for calculation. Also, the internal force, stress, and deformation result of the control section has been checked by referring to the current specification, and we get the numbers of loading vehicles, loading position, and loading efficiency. By comparing the effect value under loading and the calculation value based on calculation model, the results showed that the bearing capacity, rigidity and crack width of Chang pugang Bridge meet the specifications.

\section{Project profile}

\section{Bridge instructions}

Chang pugang Bridge is located in Tongcheng County, over Chang pugang River, the new bridge is built in the downstream of the old bridge with the distance of two meters. The new bridge was constructed with prestressed concrete hollow slab, there are 11 spans, the length of each span is 16.8 meters, the abutment of the bridge is columnar with bored pile, also, the stake is columnar with bored pile. The bridge is constructed with 0.75 meters of sidewalk, 7.0 meters of carriageway, 2.5 meters of central median, 7.0 meters of carriageway, 0.75 meters of sidewalk, 18.0 meters in total. The design load is Highway-secondary. The aim of the load test is to check the quality of the bridge and provide information for further maintenance management. The full view of Chang pugang Bridge is showed in Fig. 1.

1.2 Main technical parameters

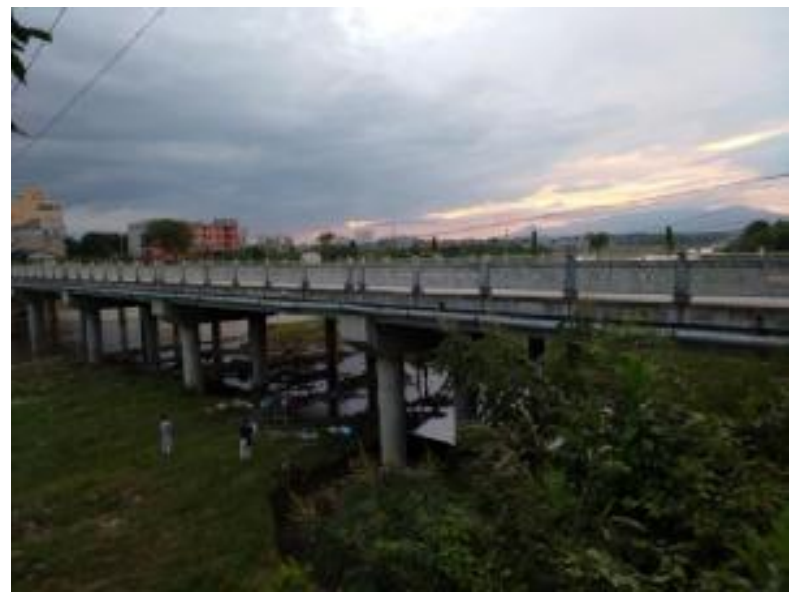

Fig. 1 The full view of Chang pugang Bridge

1) Design loading: Highway-secondary;

2) Width of bridge: 18 meters.

1.3 Main gist

1) Extension project construction drawing design of Chang pugang Bridge;

2) Experiment procedure of highway bridge ( JTG/J21-01-2015);

3) General design specification for highway bridge ( JTG D60-2015);

4) Test and assessment procedure for carrying capacity of highway bridge (JTG/T J21 -2011). 


\section{Structural simulation models}

\section{Parameters of loading vehicle}

The test load of this bridge is acquired by equivalent transformation of the worst effect of the control section under Highway-secondary loading. By calculation, two vehicle weighed $369 \mathrm{kN}$ in total is needed.

Table 1 The vehicles needed in the test

\begin{tabular}{|l|l|l|}
\hline Car Number & Weight $(\mathrm{kN})$ & Wheelbase \\
\hline 鄂L53978 & 368.8 & \multirow{2}{*}{1.4 meters +3.5 meters } \\
\cline { 1 - 2 } 赣G53520 & 367.5 & \\
\hline
\end{tabular}

\section{Structural simulation model of Civil}

In order to guarantee the accuracy of the theoretical analysis, finite element software Midas Civil is adopted in Chang pugang Bridge, upper bearing structure is analyzed according to the simplified model. Showed in Fig. 2

Based on the clause 5.4.1 and 5.4.2 in Experimental procedure of highway bridge load (JTG/ J21-01-2015), "Design load should acts as control load in experimental on acceptance load labor", "Moderate and small span bridge are girder structures mostly, and it is designed according to lateral distribution theory, the effect of largest internal force is used as control object in load test with special concern on the limitation of the loading efficiency of other beams". Lateral distribution theory is used in the analysis of Chang pugang Bridge.

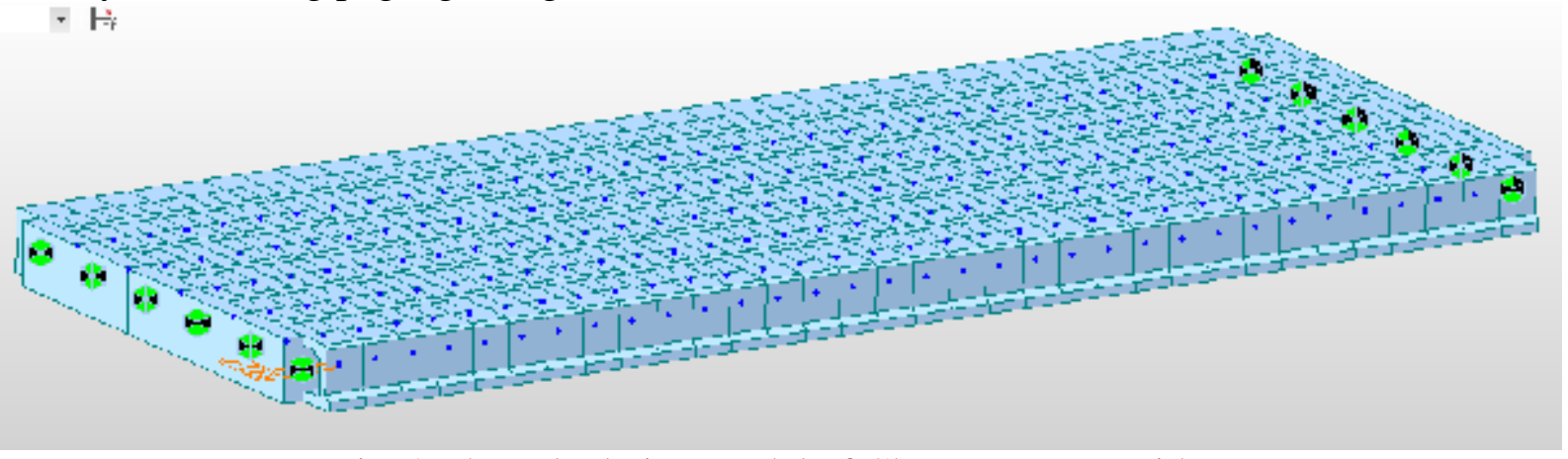

\section{Result of structural simulation}

Fig. 2 The calculation model of Chang pugang Bridge

Midas Civil is adopted in the calculation of the effect of the load. Finite element model is showed in Fig. 3, mid-span moment of each beam is showed in table 2, the internal force and deflection of the beams under medium loading working condition and unbalance loading working condition is showed in Fig. 4 Fig.5 .

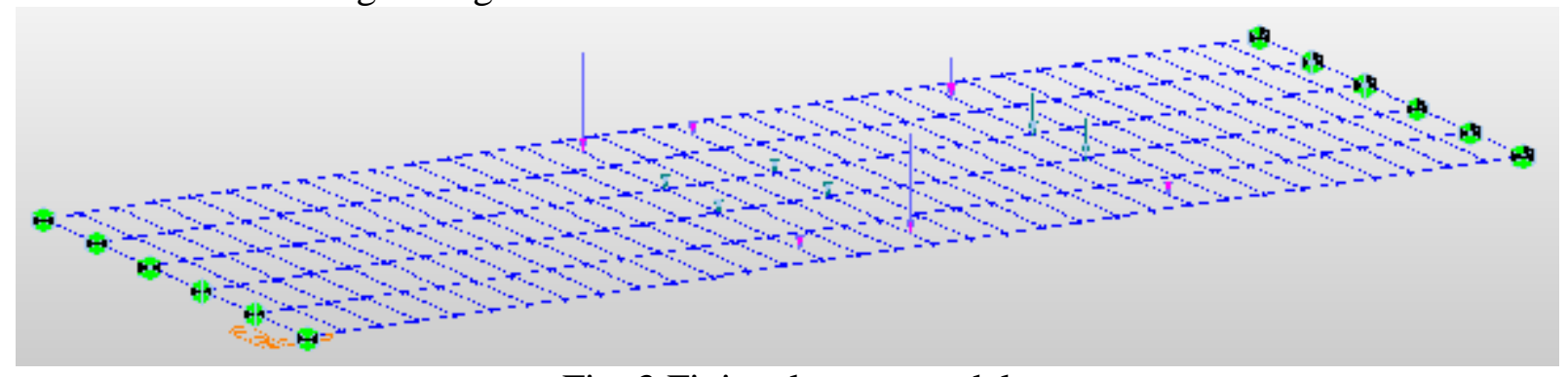

Fig. 3 Finite element model

Table 2 Mid-span moment of each beam

\begin{tabular}{|l|l|l|l|l|l|l|}
\hline Number & 1 & 2 & 3 & 4 & 5 & 6 \\
\hline Medium loading & 461.9 & 464.7 & 472.9 & 472.9 & 464.7 & 461.9 \\
\hline Unbalance loading & 490.4 & 484.3 & 479.1 & 262.8 & 451.9 & 433.5 \\
\hline
\end{tabular}




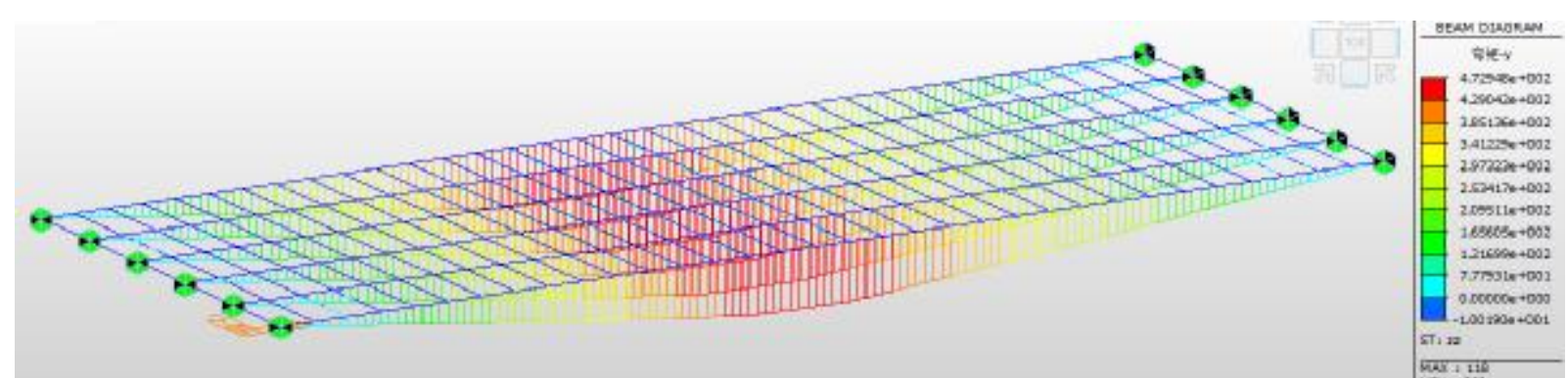

Fig. 4 Bending moment under secondary loading (medium loading)

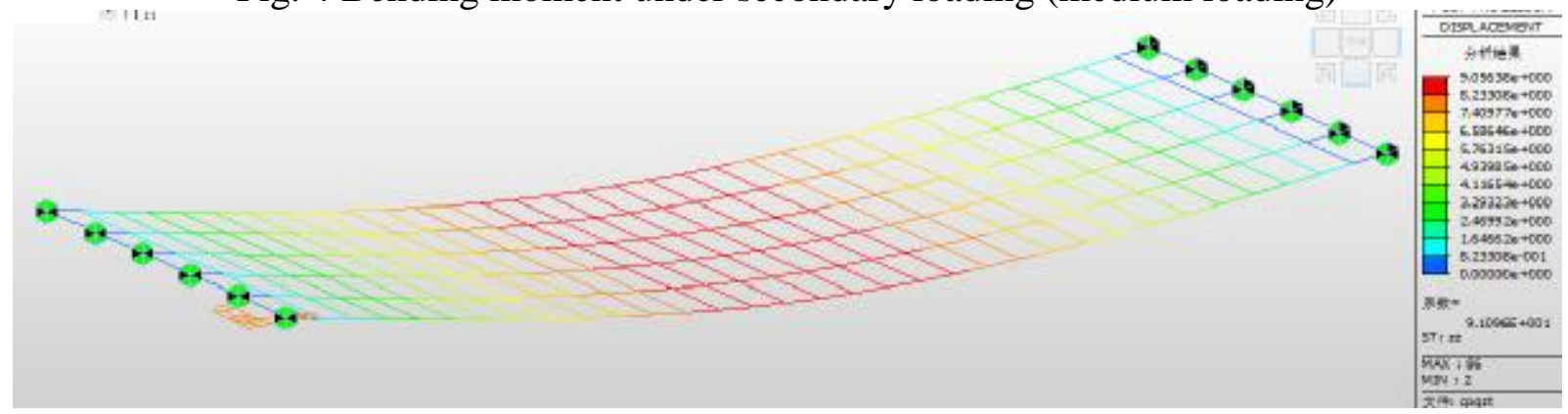

Fig. 5 Deflection under secondary loanding (medium loading)

\section{Static load conditions and load efficiency factor}

\section{Static load conditions}

Two types of static load conditions is adopted.

One: the max bending moment of mid-span section under medium loading working condition;

Two: the max bending moment of mid-span section under unbalance loading working condition.

According to the clause in Experimental procedure of highway bridge load (JTG/ J21-01-2015), the efficiency coefficient of static loading test in completion acceptance is between 0.85 and $1.05\left(0.85 \leq \eta_{q} \leq 1.05\right)$.

$$
\eta_{q}=\frac{s_{s}}{s(1+\mu)}
$$

$S_{s}:$ The effect value of the internal force of control section under test load;

$S$ : The calculated value of the internal force of control section under largest load; $\mu$ : Impact coefficient.

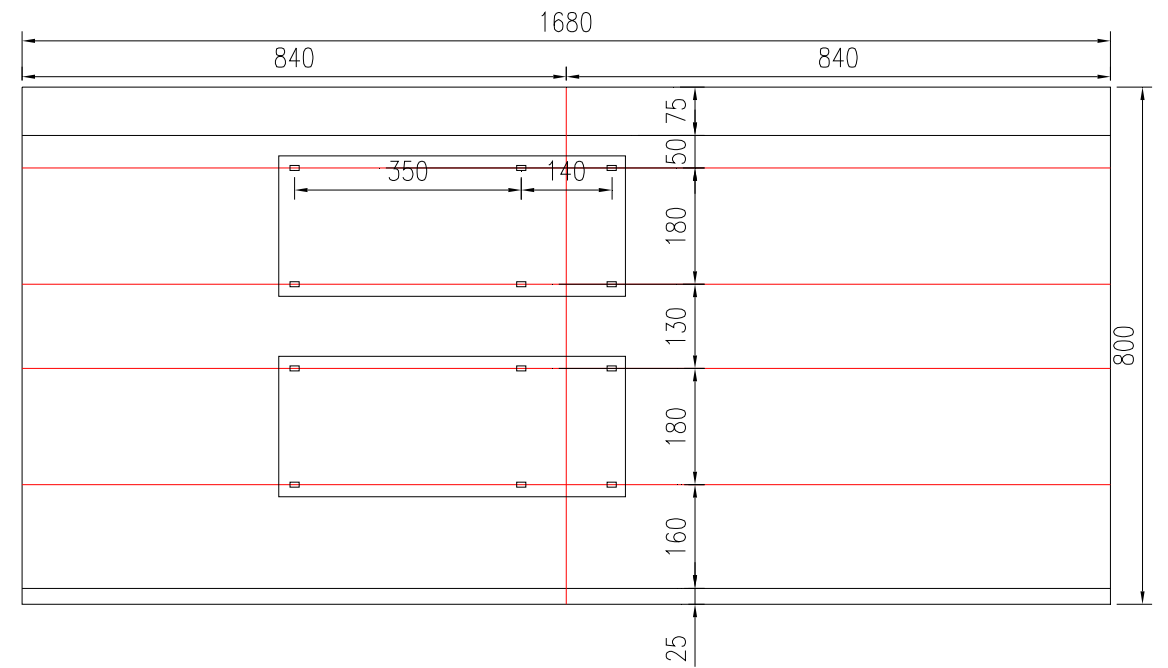

Fig. 6 Lateral distribution of vehicles under unbalance loading 


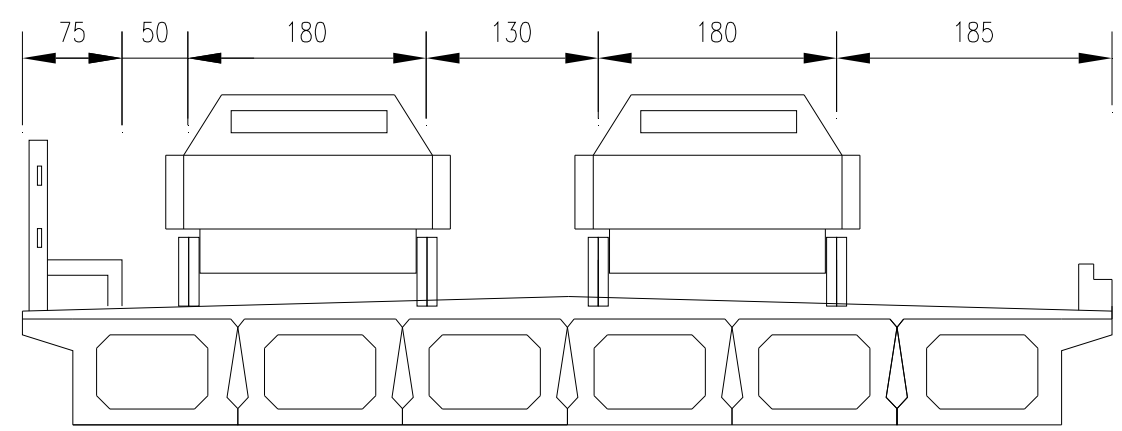

Fig. 7 Complanate distribution of vehicles under unbalance loading

\section{Efficiency coefficient of experimental condition}

Efficiency coefficient under medium loading working condition is 0.922 , efficiency coefficient under unbalance loading working condition is 0.935 , all of them are between 0.85 and 1.05 , which guarantees the validity of the test.

\section{Conclusions}

1 Efficiency coefficient

Efficiency coefficient under medium loading working condition is 0.922 , efficiency coefficient under unbalance loading working condition is 0.935 , which meet the clause in (Experimental procedure of highway bridge load (JTG/J21-01-2015), the efficiency coefficient in static load test should be controlled between 0.85 and 1.05 .

2 Bearing capacity

Strain checking coefficient of each working condition is between 0.20 and 0.50 , which meet the clause in Experimental procedure of highway bridge load (JTG/J21-01-2015) in general, strain checking coefficient of each working condition is between 0.60 and 0.90 . it shows that the intensity is satisfied.

\section{Rigidity}

Deflection checking coefficient of each working condition is between 0.28 and 0.34 , which meet the clause in Experimental procedure of highway bridge load (JTG/J21-01-2015) in general, deflection checking coefficient of each working condition is between 0.70 and 1.00 . The rigidity meets the design value.

4 Cracks

After the test, the old cracks do not expand, and new crack has not been found.

\section{Acknowledgements}

The author gratefully acknowledge financial supported by the Science Foundation of Hubei Provincial Transportation Hall (No, 2014-721-9-1).

\section{References}

[1] Lichu Fan. Bridge Engineering [M]. Beijing: China Communication Press, 1990

[2] Jianshu Ye. Principle of structure design [M]. Beijing: China Communication Press, 2005

[3] Binchu Yang. Finite element [M]. Xi'an: Xii University Press, 1996

[4] Weijian Yi. Concrete structure test and theoretical research [M]. Beijing: Science Press, 2012 